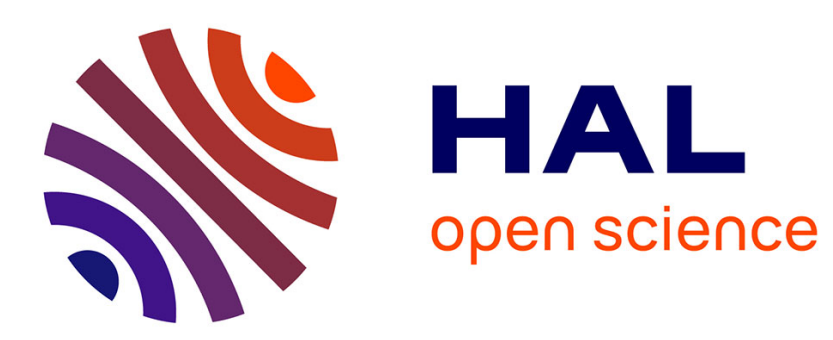

\title{
Numerical simulations of a middle gap turbulent Taylor-Couette-Poiseuille flow
}

Romain Oguic, Stéphane Viazzo, Sébastien Poncet

\section{To cite this version:}

Romain Oguic, Stéphane Viazzo, Sébastien Poncet. Numerical simulations of a middle gap turbulent Taylor-Couette-Poiseuille flow. Direct and Large-Eddy Simulation 9, Apr 2013, Dresden, Germany. hal-01098564

\section{HAL Id: hal-01098564 https://hal.science/hal-01098564}

Submitted on 26 Dec 2014

HAL is a multi-disciplinary open access archive for the deposit and dissemination of scientific research documents, whether they are published or not. The documents may come from teaching and research institutions in France or abroad, or from public or private research centers.
L'archive ouverte pluridisciplinaire HAL, est destinée au dépôt et à la diffusion de documents scientifiques de niveau recherche, publiés ou non, émanant des établissements d'enseignement et de recherche français ou étrangers, des laboratoires publics ou privés. 


\title{
Numerical simulations of a middle gap turbulent Taylor-Couette-Poiseuille flow
}

\author{
R. Oguic, S. Viazzo, and S. Poncet
}

\section{Introduction}

Turbulent flows in an opened Taylor-Couette system with an axial throughflow is studied here by the means of large eddy simulations. The ultimate industrial application is the effective cooling of the rotor-stator gap of an electrical motor. The recent review of Fénot et $a l$. [4] pointed out the lack of reliable data in such configurations for the hydrodynamic fields and most of all for the thermal one. Among others, Nouri and Whitelaw [5] and Escudier and Gouldson [3] provided detailed velocity measurements in very elongated middle-gap cavities. In a recent paper, Poncet et al. [6] used a second-order turbulence model assuming the flow as being steady and axisymmetric, whose results compared quite well with the ones of [3]. Little is known about the near-wall turbulent structures when an axial Poiseuille is superimposed. We can cite the numerical work of Chung and Sung [2], who highlighted the destabilization of the near-wall turbulent structures due to the rotation of the inner wall giving rise to an increase of sweep and ejection events. The main objective of the present work is to validate the present LES approach against reliable data available in the literature $[2,5]$, which can be seen as a first step towards the simulation of the real flow conditions.

\section{Geometrical configuration and flow control parameters}

The fluid is confined between two concentric cylinders of height $h$ (see Fig.1). The inner cylinder of radius $R_{1}$ is rotating at the rate $\Omega$, while the outer one of radius $R_{2}$ is stationary. The cavity may be characterized by two geometrical parameters:

S. Viazzo $\cdot$ S. Poncet $\cdot$ R. Oguic

Laboratoire M2P2 UMR 7340 CNRS - Aix-Marseille Université, Marseilles - France, e-mail:

\{viazzo, poncet, oguic\} $a 13 \mathrm{~m}$.univ-mrs.fr 
its aspect ratio $\Gamma=h /\left(R_{2}-R_{1}\right)$ and its radius ratio $\eta=R_{1} / R_{2}$, fixed here to 9 and 0.5 respectively. An axial throughflow is imposed within the gap at a constant inlet axial velocity $W_{m}$. The hydrodynamic fields are governed by the rotational and bulk Reynolds numbers defined as $R e_{\Omega}=\Omega R_{1}\left(R_{2}-R_{1}\right) / v$ and $R e_{Q}=2 W_{m}\left(R_{2}-\right.$ $\left.R_{1}\right) / v=8900$ ( $v$ the fluid kinematic viscosity). The rotational Reynolds number may vary such that the rotation parameter $N=\Omega R_{1} / W_{m}$ will take the values 0.2145 , 0.429 and 0.858 .

\section{Numerical method}

The numerical method is based on the work of Abide and Viazzo [1], who developed a 2D compact fourth-order projection decomposition method in cartesian coordinates. It has been recently validated in the case of turbulent rotor-stator flows in cylindrical coordinates by Viazzo et al. [7]. The time advancement is second order accurate and based on the explicit Adams-Bashforth scheme for the convective terms and an implicit backward Euler scheme for the viscous ones. The derivatives are approximated using fourth order compact formula in the radial and axial directions. The time splitting scheme is an improved projection method ensuring the incompressibility at each time step. The projection decomposition method is based on a direct non-overlapping multidomain Helmholtz/Poisson solver, which provides the solution of each Helmholtz/Poisson problem. The multidomain solver ensures

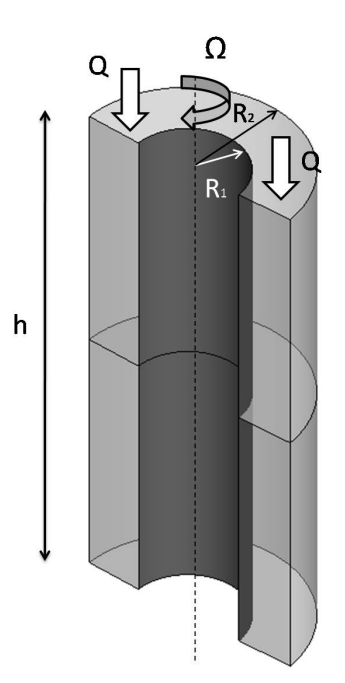

(a)

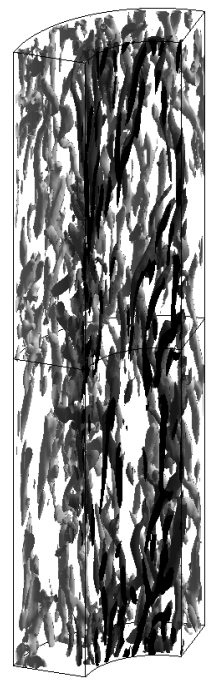

(b)

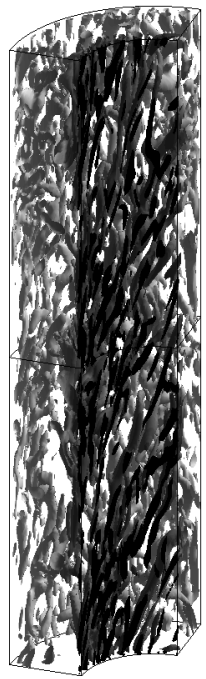

(c)

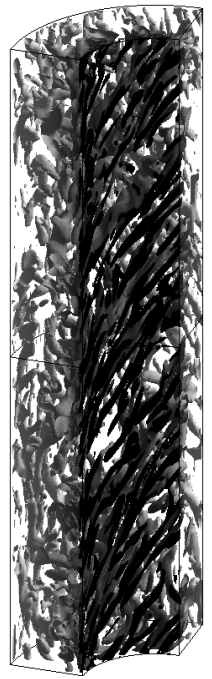

(d)

Fig. 1 (a) Schematic representation of the Taylor-Couette-Poiseuille system with relevant notations; Isosurfaces of the Q-criterion $(Q=0.5$ ) for (b) $N=0.2145$, (c) $N=0.429$ and (d) $N=0.858$ 
the continuity of the solution and its first normal derivative across the conforming interface using an influence matrix technique. Conservation equations are solved using a Fourier approximation in the homogeneous tangential direction. The problem is thus reduced at each time step to a set of two-dimensional Helmholtz equations. Periodic boundary conditions are applied in the axial and circumferential directions and no-slip boundary conditions are imposed on the walls. A dynamic Smagorinsky model is used as subgrid-scale modelling.

Table 1 Numerical details and mean flow parameters. The number of mesh points $N_{r}$ in the radial direction is fixed to 65 in all calculations. Comparisons with the LES of [2]

\begin{tabular}{lllllll}
\hline Parameters [2] & present & [2] & present & [2] & present \\
\hline$N$ & 0.2145 & 0.2145 & 0.429 & 0.429 & 0.858 & 0.858 \\
$\Delta z_{i}^{+}$ & 22.95 & 21.36 & 16.8 & 15.87 & 9.93 & 9.31 \\
$\Delta z_{o}^{+}$ & - & 19.4 & - & 13.41 & - & 7.05 \\
$\Delta r_{i}^{+}$ & 0.27 & 0.47 & 0.3 & 0.33 & 0.35 & 0.63 \\
$\Delta r_{o}^{+}$ & 0.23 & 0.43 & 0.24 & 0.45 & 0.26 & 0.47 \\
$\left(R_{1} \Delta \theta\right)^{+}$ & 8.01 & 7.34 & 8.8 & 8.22 & 10.4 & 9.7 \\
$\left(R_{2} \Delta \theta\right)^{+}$ & 13.86 & 13.33 & 14.34 & 13.9 & 15.8 & 14.7 \\
$\left(N_{\theta}, N_{z}\right)$ & $(128,128)$ & $(128,130)$ & $(128,192)$ & $(128,194)$ & $(128,384)$ & $(128,386)$ \\
$\delta t(s)$ & - & $9 \times 10^{-3}$ & - & $7 \times 10^{-3}$ & - & $4 \times 10^{-3}$ \\
$\mathrm{CFL}$ & - & 0.2 & - & 0.22 & - & 0.25 \\
$\mathrm{CPU}$ time & - & $5.2(\mathrm{~s} / \mathrm{it})$ & - & $9(\mathrm{~s} / \mathrm{it})$ & - & $25.5(\mathrm{~s} / \mathrm{it})$ \\
$C_{f, i}$ & $8.91 \times 10^{-3}$ & $8.91 \times 10^{-3}$ & $9.86 \times 10^{-3}$ & $1.08 \times 10^{-2}$ & $1.2 \times 10^{-2}$ & $1.58 \times 10^{-2}$ \\
$C_{f, o}$ & - & $7.44 \times 10^{-3}$ & - & $8.05 \times 10^{-3}$ & - & $9.05 \times 10^{-3}$ \\
$\operatorname{Re}_{\tau, i}$ & 163.21 & 149.53 & 179.24 & 167.52 & 211.85 & 197.51 \\
$\operatorname{Re}_{\tau, o}$ & 141.19 & 135.8 & 146.1 & 141.6 & 160.91 & 149.71 \\
\hline
\end{tabular}

All the numerical details used in the present work are summed in Table 1 and compared to the LES calculations of Chung and Sung [2]. It is noticeable that the domain is decomposed into 2 subdomains in the axial direction. In order to save computational ressources, the solution is calculated by assuming a $\pi / 2$-periodicity, though the grid resolutions are given for a $\pi$-periodicity to enable direct comparisons with [2]. After reaching the statistical convergence (about $2000 \mathrm{~s}$ of computed physical time), the mean and rms quantities are sampled the last $80 \mathrm{~s}$ in each case and averaged both in the tangential and axial directions. All the calculations have been performed on the M2P2 cluster composed of 2 xeon quadcore $3 \mathrm{GHz}$.

\section{Results}

The present LES results are compared to the velocity measurements of Nouri and Whitelaw [5] and to the LES of Chung and Sung [2] for three values of the rotation parameters $N=0.2145,0.429$ and 0.858 . 


\subsection{Flow structures}

To highlight the presence of near-wall structures, Figures $2 b-d$ present isovalues of the $\mathrm{Q}$-criterion colored as a function of the radius. Whatever the value of the rotation parameter $N$, coherent structures are well aligned with the axial flow along the stator. Rotation of the inner cylinder tilts the coherent structures appearing as spiral rolls and forming a negative angle (as they roll up in the opposite sense of the rotor) with the axial direction. These spirals are not observed in the LES results of Chung and Sung [2] for $N=0.429$.

\subsection{Mean flow field}

Figure 2 shows the radial distribution of the mean axial and tangential velocity components normalized respectively by $W_{m}$ and $\Omega R_{1}$. The axial velocity is weakly influenced by the rotation parameter $N$ and the profile resembles the turbulent Poiseuille profile usually observed in pipe flows. The tangential velocity profile is similar to the Batchelor profile observed in interdisk rotor-stator flows with two thin boundary layers developed on each disk separated by a central region, where $V_{\theta}$ slightly decreases with the radius. The present results are in good agreement with the experimental data of Nouri and Whitelaw [5] for $N=0.429$ and improve the previous ones of [2]. The variation of $V_{\theta}$ with $1 / r$ is recovered confirming that the angular momentum is almost constant along a radius. The average friction coefficients $C_{f}$ and the friction Reynolds numbers $R e_{\tau}$ obtained along both cylinders compare very favorably to the LES results of Chung and Sung [2] as listed in Table 1.

\subsection{Turbulent flow field}

The radial variations of the $R_{\theta \theta}$ and $R_{r z}$ components of the Reynolds stress tensor normalized by $W_{m}^{2}$ are displayed in Figure 2 . Velocity fluctuations are particularly higher for the tangential component with very intense peak values close to the walls. For the two other normal components (not shown here), the same behavior is observed with almost constant turbulence intensities within the gap, which vanish towards the cylinders. As expected, increasing the rotation parameter $N$ (ie the rotation rate $\Omega$ ) induces higher turbulence levels especially for the radial and tangential components. Furthermore, the distributions of the normal components are asymmetric, which may be attributed to the destabilizing effect of the centrifugal force. The $R_{r z}$ shear component of the Reynolds stress tensor is shown in Figure 2. When $N$ increases, the shear stresses increase in strength too. A good quantitative agreement has been obtained between our present LES results and the data of Nouri and Whitelaw [5]. It slightly improves the LES results of [2] highlighting the importance of the high order schemes. 
Figure 3 presents the anisotropy invariant map for the Reynolds stress tensor. It is noticeable that the present LES results respect the realizability diagram of Lumley. As expected, turbulence is mainly at two-component close to the cylinders and tends to the isotropic state within the center of the gap. There is only a weak effect of the rotation parameter, the flow getting more isotropic in the center region for high $N$ values. Our results and the ones of [2] do not support their conclusions about a "disk-like" state at mid-radius. Even if the third invariant gets weakly negative, all the normal components of the Reynolds stress tensor are indeed all of the same order.

\section{Conclusion}

The LES code developed by Viazzo et al. [7] has been extended here to its multidomain version using the methodology described in [1]. This new approach has
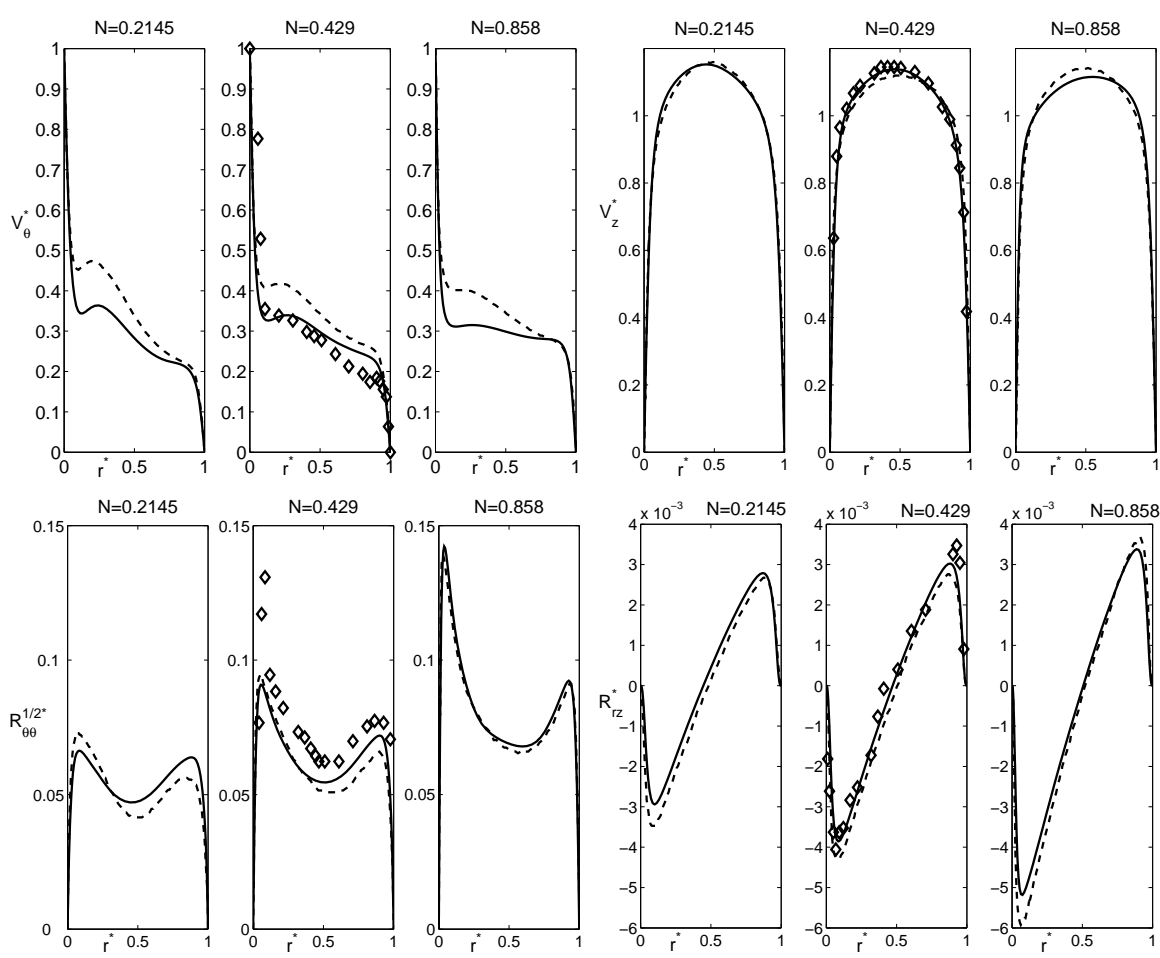

Fig. 2 Radial distributions of the mean tangential and axial velocity components and of the two components of the Reynolds stress tensor for the three values of $N$. Present LES (straight lines), LES of Chung and Sung [2] (dashed lines) and measurements of Nouri and Whitelaw [5] (symbols) 
been fully validated against the velocity measurements of Nouri and Whitelaw [5] and the numerical results of Chung et $a l$. [2] in an opened Taylor-Couette system with an axial throughflow. Near-wall structures appearing as elongated spiral rolls are observed along the inner cylinder. A very good agreement has been obtained for both the mean and turbulent fields and for the three values of the rotation parameter considered highlighting the importance of the order of the spatial schemes. Some numerical developments are still required to simulate the whole problem of the effective cooling in the rotor-stator gap of an electrical motor.

\section{References}

1. Abide, S., Viazzo, S.: A 2D compact fourth-order projection decomposition method. J. Comp. Phys. 206, 252-276 (2005)

2. Chung, S.Y., Sung, H.J.: Large-eddy simulation of turbulent flow in a concentric annulus with rotation of an inner cylinder. Int. J. Heat Fluid Flow 26, 191-203 (2005)

3. Escudier, M.P., Gouldson, I.W.: Concentric annular flow with centerbody rotation of a Newtonian and a shear-thinning liquid. Int. J. Heat Fluid Flow 16, 156-162 (1995)

4. Fénot, M., Bertin, Y., Dorignac, E., Lalizel, G.: A review of heat transfer between concentric rotating cylinders with or without axial flow. Int. J. Therm. Sci. 50, 1138-1155 (2011)

5. Nouri, J.M., Whitelaw, J.H.: Flow of newtonian and non-newtonian fluids in a concentric annulus with rotation of the inner cylinder. J. Fluid Eng. 116, 821-827 (1994)

6. Poncet, S., Haddadi, S., Viazzo, S.: Numerical modeling of fluid flow and heat transfer in a narrow Taylor-Couette-Poiseuille system. Int. J. Heat Fluid Flow 32 (1), 128-144 (2011)

7. Viazzo, S., Poncet, S., Serre, E., Randriamampianina, A., Bontoux, P.: High-order Large Eddy Simulations of confined rotor-stator flows. Flow, Turbulence \& Combustion 88 (1-2), 63-75 (2012)

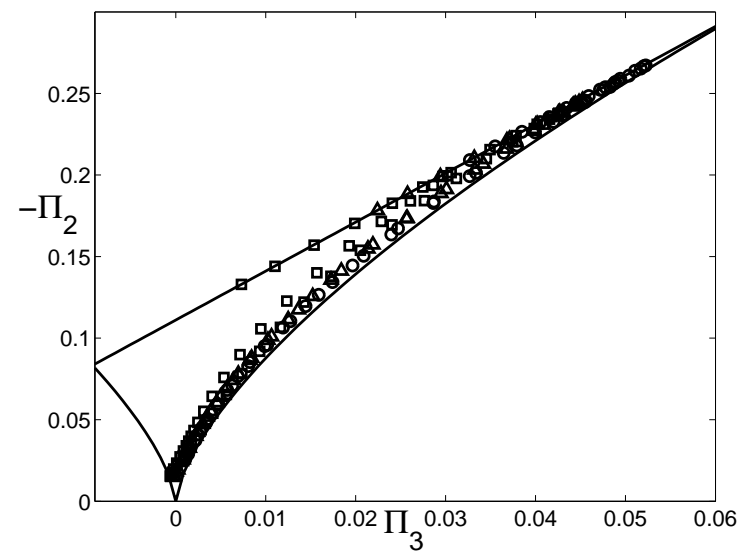

Fig. 3 Anisotropy invariant map for the Reynolds stress tensor: $N=0.2145$ (circles), $N=0.429$ (triangles) and $N=0.858$ (squares) 